\title{
Theoretical Re-evaluations of Scaling Relations between SMBHs and Their Host Galaxies-1. Effect of Seed BH Mass
}

\author{
Hikari Shirakata ${ }^{1 *}$, Toshihiro Kawaguchi ${ }^{2}$, Takashi Okamoto ${ }^{1}$, Ryu Makiya ${ }^{3,4}$, \\ Tomoaki Ishiyama ${ }^{5}$, Yoshiki Matsuoka ${ }^{6}$, Masahiro Nagashima ${ }^{7}$, Motohiro Enoki $^{8}$, \\ Taira Oogi ${ }^{3}$ and Masakazu A. R. Kobayashi ${ }^{9}$
}

\begin{abstract}
${ }^{1}$ Department of Cosmosciences, Graduate School of Science, Hokkaido University, Sapporo, Japan, ${ }^{2}$ Department of Economics, Management and Information Science, Onomichi City University, Onomichi, Japan, ${ }^{3}$ Kavli Institute for the Physics and Mathematics of the Universe, Todai Institutes for Advanced Study, University of Tokyo, Kashiwa, Japan, ${ }^{4}$ Max-Planck-Institut fur Astrophysik, Garching, Germany, ${ }^{5}$ Institute of Management and Information Technologies, Chiba University, Chiba, Japan, ${ }^{6}$ Research Center for Space and Cosmic Evolution, Ehime University, Matsuyama, Japan, ${ }^{7}$ Faculty of Education, Bunkyo University, Koshigaya, Japan, ${ }^{8}$ Faculty of Business Administration, Tokyo Keizai University, Kokubunji, Japan, ${ }^{9}$ Faculty of Natural Sciences, National Institute of Technology, Kure College, Kure, Japan
\end{abstract}

OPEN ACCESS

Edited by:

Mauro D'Onofrio

University of Padua, Italy

Reviewed by:

Jaime Perea,

Instituto de Astrofísica de Andalucía

(CSIC), Spain

Vyacheslav Ivanovich Dokuchaev, Institute for Nuclear Research (RAS),

Russia

*Correspondence: Hikari Shirakata shirakata@astro1.sci.hokudai.ac.jp

Specialty section:

This article was submitted to

Milky Way and Galaxies,

a section of the journal

Frontiers in Astronomy and Space

Sciences

Received: 10 July 2017 Accepted: 08 September 2017 Published: 21 September 2017

Citation:

Shirakata H, Kawaguchi T, Okamoto T, Makiya R, Ishiyama T, Matsuoka $Y$, Nagashima M, Enoki M, Oogi T and Kobayashi MAR (2017) Theoretical Re-evaluations of Scaling Relations between SMBHs and Their Host Galaxies - 1. Effect of Seed BH Mass

Front. Astron. Space Sci. 4:15. doi: 10.3389/fspas.2017.00015
We use a semi-analytic model of galaxy formation and investigate how the mass of a seed black hole affect the scaling relation between black hole mass and bulge mass at $z \sim 0$. When the mass of the seed is set at $10^{5} M_{\odot}$, we find that the model results become inconsistent with recent observational results of the scaling relation for dwarf galaxies. On the other hand, when we set seed black hole mass as $10^{3} M_{\odot}$ or as randomly chosen value within a $10^{3-5} M_{\odot}$ range, we find the results are consistent with observational results including the dispersion. We also find that black hole mass - bulge mass relations for less massive bulges at $z \sim 0$ put stronger constraints on the seed $\mathrm{BH}$ mass than the relations at higher redshifts.

Keywords: galaxies, active galactic nuclei, bulge, galaxy formation, statistics

\section{INTRODUCTION}

Many observations (e.g., Kormendy and Richstone, 1995; Magorrian et al., 1998; Häring and Rix, 2004; McConnell and Ma, 2013) have suggested that the mass of supermassive black holes $\left(M_{\mathrm{BH}}\right)$ correlates with the properties of their host galaxies such as stellar mass of bulges $\left(M_{\text {bulge }}\right)$ at $z \sim 0$. This $M_{\mathrm{BH}}-M_{\text {bulge }}$ relation might suggest that supermassive black holes (SMBHs) would have co-evolved with their host galaxies.

SMBHs grow to the current mass $\left(\gtrsim 10^{6} M_{\odot}\right)$ from their initial mass. The initial mass and its distribution have been debating. Although, there are many theoretical suggestions of formation mechanism and mass of seed BHs (e.g., Begelman et al., 2006), we cannot obtain what is the dominant mechanism by comparing theoretical models with observations since seed $\mathrm{BHs}$ are not observable directly.

Here, we focus on the $M_{\mathrm{BH}}-M_{\text {bulge }}$ relation for galaxies with bulge mass is less than $10^{10} M_{\odot}$ to get the constraints on mass of seed BHs. This paper is a summary of Shirakata et al. (2016) in which we investigate the effect of the seed BHs' mass on model predictions of $M_{\mathrm{BH}}-M_{\text {bulge }}$ relation at $M_{\text {bulge }} \lesssim 10^{10} M_{\odot}$ by using an semi-analytic model of galaxy formation (hereafter SA model). In section 2 we briefly review the SA model we used. Section 3 includes the main results. Finally, in section 4 , we summarize this review and briefly mention future prospects. 


\section{MODELS}

We use a revised version of an SA model, "New Numerical Galaxy Catalogue" ( $v^{2}$ GC; Makiya et al., 2016, hereafter M16), where the models related to the SMBH and AGNs are described in Enoki et al. (2003), Enoki et al. (2014), and Shirakata et al. (2015). We consider star formation in galactic disk and bulge, mergers of galaxies, atomic gas cooling, gas heating by UV feedback and feedbacks via supernovae and AGNs, and the growth of SMBHs by coalescence and gas accretion from their host galaxies.

Merging histories of dark matter halos are calculated from state-of-the-art cosmological $\mathrm{N}$-body simulations (Ishiyama et al., 2015). The cosmological simulations have a high mass resolution and large volume compared to previous simulations (e.g., mass resolution is roughly four times better than those of Millennium simulations, Springel et al., 2005). Here we employ a simulation with $L=70.0\left[h^{-1} \mathrm{Mpc}\right]$ of box size and $512^{3}$ particles, which corresponds to $M_{\min }=2.20 \times 10^{8}\left[h^{-1} M_{\odot}\right]$ of minimum halo mass.

We assume a $\Lambda$ CDM universe which have the following parameters: $\Omega_{0}=0.31, \lambda_{0}=0.69, \Omega_{\mathrm{b}}=0.048, \sigma_{8}=0.83$, $n_{\mathrm{s}}=0.96$, and a Hubble constant of $H_{0}=100 \mathrm{~h} \mathrm{~km} \mathrm{~s} \mathrm{Mpc}^{-1}$, where $h=0.68$ (Planck Collaboration et al., 2014).

\subsection{Setting of Seed Black Holes}

We place a seed $\mathrm{BH}$ soon after the time of a galaxy formation. We present results with $M_{\mathrm{BH} \text {,seed }}=10^{3} M_{\odot}$ (hereafter "light seed model") where $M_{\mathrm{BH} \text {,seed }}$ is the seed $\mathrm{BH}$ mass, and $10^{5} \mathrm{M}_{\odot}$ ("massive seed model"). In addition, we employ the model in which $M_{\mathrm{BH} \text {,seed }}$ takes uniformly random values in the logarithmic scale in the range of $3 \leq \log \left(M_{\mathrm{BH} \text {,seed }} / M_{\odot}\right) \leq 5$ (hereafter "random seed model").

\subsection{Summary of Bulge and SMBH Growth Model}

We assume that the bulge grows via starbursts and the migration of disk stars. Starbursts are triggered by mergers of galaxies (major and minor) or disk instability. The model of merger driven bulge formation in $v^{2}$ GC is based on Hopkins et al. (2009). We consider that mergers of galaxies occur both by dynamical friction (central-satellite merger) and random collision (satellitesattelite merger). We also introduce the spheroid formation by disk instability following Mo et al. (1998) and Cole et al. (2000). In both cases, the gas supplyed from galactic disk to the bulge is completely exhausted by a starburst and fueling onto their central SMBHs.

SMBHs in $v^{2}$ GC are mainly grown by gas accretion from their host galaxy. When a starburst occurs in a bulge, a part of cold gas gets accreted by the SMBH :

$$
M_{\mathrm{acc}}=f_{\mathrm{BH}} \Delta M_{*, \text { burst }},
$$

where $M_{\text {acc }}$ is the cold gas mass accreted onto the $\mathrm{SMBH}$, which is assumed to be proportional to the stellar mass formed by a current starburst, $\Delta M_{* \text {,burst }}$. Here we set $f_{\mathrm{BH}}=0.01$. SMBHs also grow via coalescence of $\mathrm{BH}$ which occurs with mergers of host galaxies. For simplicity, we assume BHs merge instantaneously when their host galaxies merge.

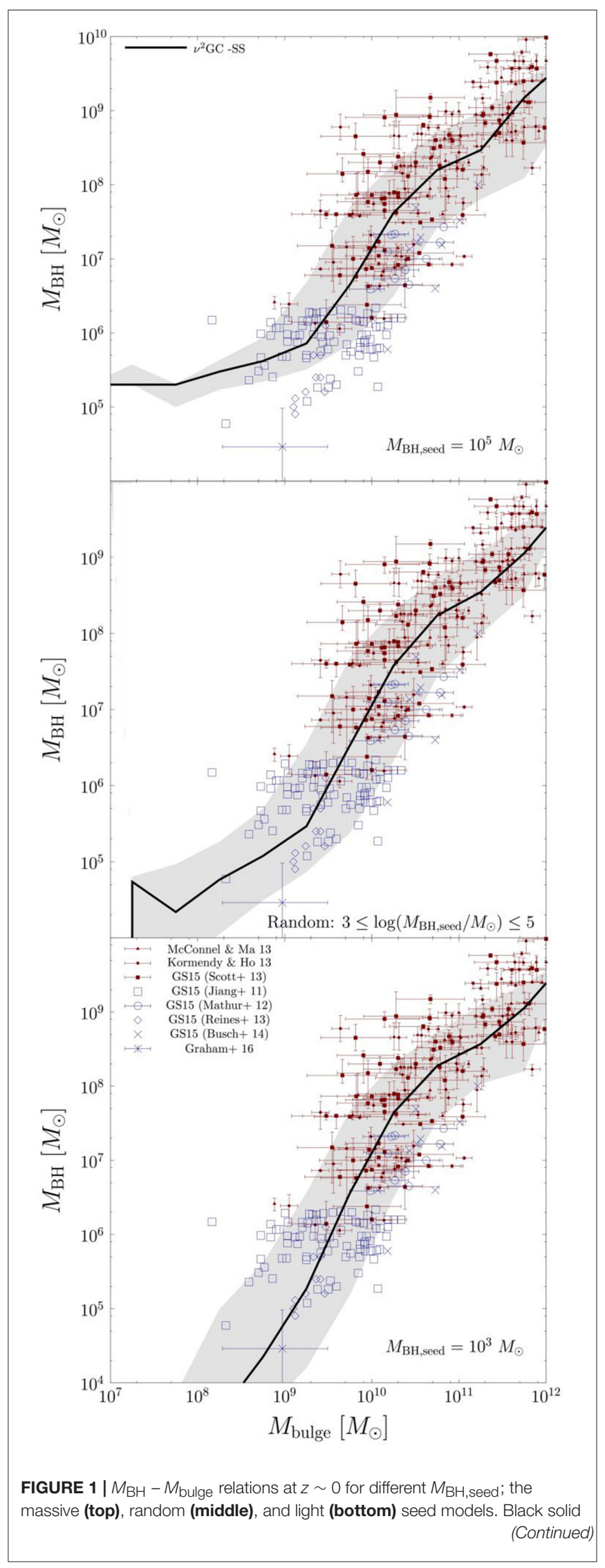




\section{FIGURE 1 | Continued}

lines track the median, and shaded regions indicate 10-90 percentile of the models of the model result. Red filled symbols indicate observational results obtained from McConnell and Ma (2013), Kormendy and Ho (2013), and GS15 ${ }^{3}$ (triangles, diamonds, and squares, respectively). Blue open symbols are AGN sample obtained from GS15, (see the text for more details). Blue asterisks correspond LEDA 87300 (Baldassare et al., 2015; Graham et al., 2016).

\section{RESULTS}

Figure 1 shows the main result which depicts the $M_{\mathrm{BH}}-$ $M_{\text {bulge }}$ relation at $z \sim 0$ obtained from the model and observations. Each panels correspond to the results of massive seed model (top), random seed model (middle), and light seed model (bottom), respectively. Red solid lines represents the model result, blue and green points represents the observational data.

We find all of the models reproduce the relation at $M_{\text {bulge }} \gtrsim$ $10{ }^{10} M_{\odot}$, while the massive seed model has an inconsistency in the observational results for less massive galaxies $\left(M_{\text {bulge }} \lesssim\right.$ $\left.10^{10} M_{\odot}\right)$. Random and light seed models, on the other hand, provide the consistent results in the range of $M_{\mathrm{BH}} \gtrsim 10^{5.5} M_{\odot}$, with observational estimates. We thus conclude that to explain recent observational data of the $M_{\mathrm{BH}}-M_{\text {bulge }}$ relation at $z \sim 0$, seed $\mathrm{BH}$ mass should dominate with $\sim 10^{3} M_{\odot}$.

We note that since the number of samples of galaxies with $M_{\mathrm{BH}} \lesssim 10^{5.5} M_{\odot}$ (corresponds to $M_{\text {bulge }} \lesssim 10^{10} M_{\odot}$ ) are not sufficient. Observational data with the mass range are thus necessary to investigate the detailed mass distribution of the seed BHs. It is however difficult to estimate $\mathrm{BH}$ and bulge mass of less massive galaxies. We thus investigate whether the $M_{\mathrm{BH}}-$ $M_{\text {bulge }}$ relation at higher redshifts could be useful for getting further constraints on the mass of seed BHs. Figure 2 displays the ratio of the average $\mathrm{BH}$ masses in the light seed model $\left(\equiv\left\langle M_{\mathrm{BH}}\right\rangle_{3}\right)$ and those in the massive seed model $\left(\equiv\left\langle M_{\mathrm{BH}}\right\rangle_{5}\right)$, as a function of bulge masses. The difference in the seed mass significantly appears in galaxies with bulge mass below $3 \times 10^{9} \mathrm{M}_{\odot}$ at $z \sim 0,1$, and 2 . We also find that the difference becomes smaller at higher redshift for a given $M_{\text {bulge }}$. Observations of less massive bulges at $z \sim 0$ would thus be more important than at higher redshifts for investigating the mass distribution of seed BHs.

\section{SUMMARY AND FUTURE PROSPECTS}

We investigate how the mass of the seed BHs affects model predictions of the local $M_{\mathrm{BH}}-M_{\text {bulge }}$ relation by using an SA model. The results suggest that seed $\mathrm{BHs}$ with as massive as $10^{5} M_{\odot}$ should not be dominant for reproducing the observed $M_{\mathrm{BH}}-M_{\text {bulge }}$ relation at $z \sim 0$ over a wide range of bulge masses down to $M_{\text {bulge }} \lesssim 10^{10} M_{\odot}$. Obtaining stronger constraints of the

\footnotetext{
${ }^{3}$ Originally obtained from Scott et al. (2013).
}

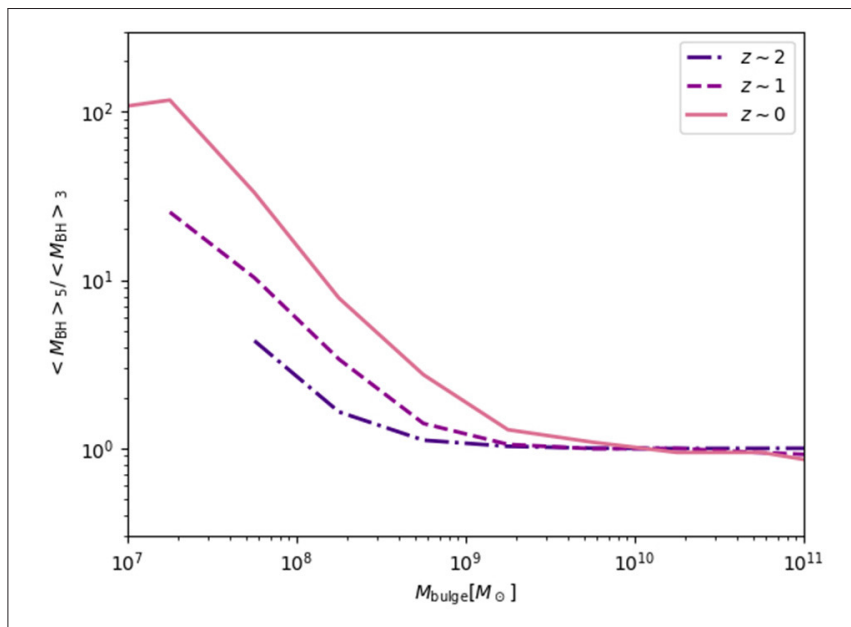

FIGURE 2 | The difference of averaged SMBH mass due to the seed BH mass at $z \sim 2$ (dash-doted line in blue), $z \sim 1$ (dashed line in purple), and $z \sim 0$ (solid line in pink) as a function of their bulge stellar mass with the $v^{2} \mathrm{GC}-\mathrm{H} 2$ simulation. The difference becomes smaller at higher redshift.

detailed mass distribution of seed BHs observations of $M_{\mathrm{BH}} \lesssim$ $10^{5.5} M_{\odot}$ would be required.

We have shown results of the local $M_{\mathrm{BH}}-M_{\text {bulge }}$ relations varying the mass of seed BHs. According to Shankar et al. (2016), $M_{\text {bulge }}$ obtained from observations could be biased in favor of larger stellar masses. If so, we might have to use $M_{\mathrm{BH}}$ - velocity dispersion relation instead of the local $M_{\mathrm{BH}}-M_{\text {bulge }}$. We leave it for future studies.

The spheroids formed through disk instability might be classified as so-called "pseudo bulges". There are some debates whether pseudo bulges and classical bulges follow the same $M_{\mathrm{BH}}$ - $M_{\text {bulge }}$ relation (e.g., Kormendy and Ho, 2013). We might need the model of the properties of pseudo bulges in the near future.

\section{AUTHOR CONTRIBUTIONS}

HS, RM, MN, ME, and MK have developed $v^{2} \mathrm{GC}$. In addition, HS analyze the output data obtained from $v^{2} \mathrm{GC}$. TK, TakO, and TaiO gave comments for the analysis. TI provides merger trees obtained from cosmological $N$-body simulations for $v^{2} \mathrm{GC}$. YM gave fruitful comments from a standpoint of AGN observations.

\section{FUNDING}

TK was supported in part by an University Research Support Grant from the NAOJ and JSPS KAKENHI (17K05389). TakO was supported by JSPS Grant-in-Aid for Young Scientists (16H01085). RM was supported in part by MEXT KAKENHI (15H05896). TI was supported by MEXT HPCI STRATEGIC PROGRAM and MEXT/JSPS KAKENHI (15K12031) and by Yamada Science Foundation. MN was supported by the Grantin-Aid (25287041 and 17H02867) from the MEXT of Japan. 


\section{REFERENCES}

Baldassare, V. F., Reines, A. E., Gallo, E., and Greene, J. E. (2015). A 50,000 $M_{\odot}$ solar mass black hole in the nucleus of RGG 118. Astrophys. J. Lett. 809:L14. doi: 10.1088/2041-8205/809/1/L14

Begelman, M. C., Volonteri, M., and Rees, M. J. (2006). Formation of supermassive black holes by direct collapse in pre-galactic haloes. Mon. Not. R. Astron. Soc. 370, 289-298. doi: 10.1111/j.1365-2966.2006.10467.x

Cole, S., Lacey, C. G., Baugh, C. M., and Frenk, C. S. (2000). Hierarchical galaxy formation. Mon. Not. R. Astron. Soc. 319, 168-204. doi: 10.1046/j.1365-8711.2000.03879.x

Enoki, M., Ishiyama, T., Kobayashi, M. A. R., and Nagashima, M. (2014). Anti-hierarchical evolution of the active galactic nucleus space density in a hierarchical universe. Astrophys. J. 794:69. doi: 10.1088/0004-637X/794/1/69

Enoki, M., Nagashima, M., and Gouda, N. (2003). Relations between galaxy formation and the environments of quasars. Publ. Astron. Soc. Jpn. 55, 133-142. doi: $10.1093 /$ pasj/55.1.133

Graham, A. W., Ciambur, B. C., and Soria, R. (2016). Does the intermediate-mass black hole in LEDA 87300 (RGG 118) follow the near-quadratic $M_{\mathrm{BH}}-M_{\text {Spheroid }}$ relation? Astrophys. J. 818:172. doi: 10.3847/0004-637X/818/2/172

Graham, A. W., and Scott, N. (2015). The (black hole)-bulge mass scaling relation at low masses. Astrophys. J. 798:54. doi: 10.1088/0004-637X/798/1/54

Häring, N., and Rix, H.-W. (2004). On the black hole mass-bulge mass relation. Astrophys. J. Lett. 604, L89-L92. doi: 10.1086/383567

Hopkins, P. F., Cox, T. J., Younger, J. D., and Hernquist, L. (2009). How do disks survive mergers? Astrophys. J. 691, 1168-1201. doi: 10.1088/0004-637X/691/2/1168

Ishiyama, T., Enoki, M., Kobayashi, M. A. R., Makiya, R., Nagashima, M., and Oogi, T. (2015). The $v^{2}$ GC simulations: quantifying the dark side of the universe in the Planck cosmology. Publ. Astron. Soc. Jpn. 67:61. doi: 10.1093/pasj/psv021

Kormendy, J., and Ho, L. C. (2013). Coevolution (or not) of supermassive black holes and host galaxies. Annu. Rev. Astron. Astrophys. 51, 511-653. doi: 10.1146/annurev-astro-082708-101811

Kormendy, J., and Richstone, D. (1995). Inward bound-the search for supermassive black holes in galactic nuclei. Annu. Rev. Astron. Astrophys. 33:581.

Magorrian, J., Tremaine, S., Richstone, D., Bender, R., Bower, G., Dressler, A., et al. (1998). The demography of massive dark objects in galaxy centers. Astron. J. 115, 2285-2305.

Makiya, R., Enoki, M., Ishiyama, T., Kobayashi, M. A. R., Nagashima, M., Okamoto, T., et al. (2016). The new numerical galaxy catalog $\left(v^{2} \mathrm{GC}\right)$ : an updated semi-analytic model of galaxy and active galactic nucleus formation with large cosmological $\mathrm{N}$ body simulations. Publ. Astron. Soc. Jpn. 68:25. doi: 10.1093/pasj/ psw005

McConnell, N. J., and Ma, C.-P. (2013). Revisiting the scaling relations of black hole masses and host galaxy properties. Astrophys. J. 764:184. doi: 10.1088/0004-637X/764/2/184

Mo, H. J., Mao, S., and White, S. D. M. (1998). The formation of galactic discs. Mon. Not. R. Astron. Soc. 295, 319-336. doi: 10.1046/j.1365-8711.1998.01227.x

Planck Collaboration, Ade, P. A. R., Aghanim, N., Alves, M. I. R., Armitage-Caplan, C., Arnaud, M., et al. (2014). Planck 2013 results. I. Overview of products and scientific results. Astron. Astrophys. 571:A1. doi: 10.1051/0004-6361/201321529

Scott, N., Graham, A. W., and Schombert, J. (2013). The supermassive black hole mass-spheroid stellar mass relation for sérsic and core-Sérsic galaxies. Astrophys. J. 768:76. doi: 10.1088/0004-637X/768/1/76

Shankar, F., Bernardi, M., Sheth, R. K., Ferrarese, L., Graham, A. W., Savorgnan, G., et al. (2016). Selection bias in dynamically-measured super-massive black holes: its consequences and the quest for the most fundamental relation. Mon. Not. R. Astron. Soc. arXiv:1603.01276.

Shirakata, H., Kawaguchi, T., Okamoto, T., Makiya, R., Ishiyama, T., Matsuoka, Y., et al. (2016). Theoretical re-evaluations of the black hole mass-bulge mass relation - I. Effect of seed black hole mass. Mon. Not. R. Astron. Soc. 461, 4389-4394. doi: 10.1093/mnras/stw1798

Shirakata, H., Okamoto, T., Enoki, M., Nagashima, M., Kobayashi, M. A. R., Ishiyama, T., et al. (2015). The impact of dust in host galaxies on quasar luminosity functions. Mon. Not. R. Astron. Soc. 450, L6-L10. doi: 10.1093/mnrasl/slv035

Springel, V., White, S. D. M., Jenkins, A., Frenk, C. S., Yoshida, N., Gao, L., et al. (2005). Simulations of the formation, evolution and clustering of galaxies and quasars. Nature 435, 629-636. doi: 10.1038/nature03597

Conflict of Interest Statement: The authors declare that the research was conducted in the absence of any commercial or financial relationships that could be construed as a potential conflict of interest.

Copyright (c) 2017 Shirakata, Kawaguchi, Okamoto, Makiya, Ishiyama, Matsuoka, Nagashima, Enoki, Oogi and Kobayashi. This is an open-access article distributed under the terms of the Creative Commons Attribution License (CC BY). The use, distribution or reproduction in other forums is permitted, provided the original author(s) or licensor are credited and that the original publication in this journal is cited, in accordance with accepted academic practice. No use, distribution or reproduction is permitted which does not comply with these terms. 